\title{
HIGH ANGULAR RESOLUTION OBSERVATIONS OF THE GAS PHASE COMPOSITION OF YOUNG STELLAR OBJECTS
}

\author{
GEOFFREY A. BLAKE \\ Division of Geological and Planetary Sciences \\ Caltech 170-25, Pasadena, CA 91125, USA
}

\begin{abstract}
Recent advances in the observational characterization of young stellar objects (YSOs) with millimeter-wave aperture synthesis arrays and (sub)millimeter single dish telescopes are reviewed. Studies of circumstellar material with arc second resolution, which have only become possible at these frequencies within the past few years and which can potentially probe all stages of the star formation process, are emphasized. Molecules that are sensitive to different routes of formation and modification are outlined, including comments about their utility in distinguishing between a variety of environments and histories in star-forming cloud cores.
\end{abstract}

\section{Introduction}

The combination of rapidly improving observational tools and increasingly sophisticated theory has provided, for the first time, a broad outline of the physical and chemical processes associated with the assembly of Sun-like stars and their attendant planetary systems (c.f. Beckwith \& Sargent 1996; Li \& Shu 1996). Many questions remain despite these impressive gains, yet the details of just how the variety of processes which accompany the collapse of molecular clouds work - and how they change with time - are crucial to understanding stellar and planet formation in general and, more specifically, to providing an understanding of the origins of the recently discovered massive extra-solar planets (c.f. Marcy \& Butler 1996).

More detailed assessments of recent theoretical advances may be found in the review by Shu in these proceedings. Here, ongoing improvements in the observational characterization of YSOs and their surroundings are highlighted, in particular the key role that observations of molecular tracers can play in understanding both the physical and chemical evolution of 
star-forming molecular cloud cores and primitive solar systems. Indeed, the tremendous range in physical conditions and size scales that are present in YSOs, where densities from $10^{4}$ to greater than $10^{13} \mathrm{~cm}^{-3}$ and temperatures of $10-10,000 \mathrm{~K}$ exist over distances from a few stellar radii to many thousands of astronomical units (AU), is perhaps best probed by molecular spectroscopy. Molecules also provide direct access to the velocity and radiation fields present in cloud cores. Finally, since chemistry controls critical physical parameters in the star formation process such as fractional ionization, a detailed understanding of the chemical composition of the gas (and dust) surrounding young stars is important and interesting in its own right. Readers interested in more detailed chemistry overviews are urged to consult other presentations in this volume and recent articles by van Dishoeck et al. (1993) and Hartquist et al. (1993).

This review will concentrate primarily on observations of molecular transitions at millimeter $(\mathrm{mm})$ and submillimeter (submm) wavelengths at high angular resolution, in particular on recent results from the California millimeter arrays. It will also include a brief discussion of dust continuum observations since they are important in establishing the overall mass and thereby the absolute chemical abundances. Imaging at (sub) $\mathrm{mm}$ wavelengths is important because young stellar systems are surrounded by large amounts of gas and dust which both participate in the formation of the star and obscure the birth process. This material is distributed in circumstellar disks with sizes of 10-100 AU and in roughly spherical envelopes extending out 100's to 1000's of AU (c.f. Shu 1995).

The envelope dominates the mass and emission in the youngest systems while the disk and star become dominant in older systems. At the early stages, the envelope can in fact have sufficient mass to obscure the disk and stellar core at optical through mid-IR wavelengths. For example, one solar mass of material with an $r^{-2}$ density distribution from 50 to $6000 \mathrm{AU}$ will exhibit a visual extinction of 200 mag over the inner 50 AU. As the YSO ages, progressively more of the total luminosity emerges at shorter wavelengths, but $\mathrm{mm}$-wave observations can probe the star formation process for at least several million years (Sargent 1996).

Once visible at shorter wavelengths, the tools of IR spectroscopy can be applied. Several critical molecular species such as $\mathrm{H}_{2}, \mathrm{CO}_{2}, \mathrm{CH}_{4}$, and $\mathrm{C}_{2} \mathrm{H}_{2}$ can, in fact, only be observed in the IR. Some of these species have been previously observed with ground-based telescopes (Lacy et al. 1991), but the full power of this approach is revealed in the pioneering ISO observations presented in these proceedings. Though there is insufficient space to cover the ground-based and ISO spectroscopy here, we note that mmwave and IR observations are highly complementary, and together provide stronger constraints on stellar and planetary formation mechanisms than 
either can alone. In what follows, we first present high resolution mm-wave observations of Orion KL to illustrate the range of important physical and chemical processes at work in star-forming cores, and then move on to a discussion of the three important components of isolated, low mass YSOs: the outflow(s), dynamically infalling envelope, and circumstellar accretion disk. Rather than present a large range of observations for each category, we present only a result or two as illustrations and refer the reader to more detailed articles in the refereed literature.

\section{Orion $\mathrm{KL}$ and high mass star formation}

The Orion KL region is one of the best studied areas of massive star formation in the Galaxy. Its close proximity and extraordinary brightness have enabled pioneering observations, that, historically, have played a pivotal role in the understanding of more distant massive YSOs (see Genzel \& Stuzki 1989). Early IR studies revealed numerous objects (Wynn-Williams et al. 1984), while molecular emission maps delineated a rich chemistry with kinematic variations that pointed to a wealth of unresolved sub-structure (Plambeck et al. 1982). The continued development of high angular resolution techniques in the IR and radio has led to ever more detailed images of Orion KL (Dougados et al. 1993; Menten \& Reid 1995). With this perspective of 20 years experience, we can appreciate that the complexity of massive star formation regions is a necessary outcome of the gregarious and destructive nature of massive stars.

As noted above, it is only recently that imaging at $<1^{\prime \prime}$ resolution has become possible at (sub)mm-wavelengths. It is in precisely these regions, however, where single dish spectral line survey work has been most rewarding (Johansson et al. 1984; Blake et al. 1987), and where (sub)arc second imaging would provide unique views of the intimate association between the physics and chemistry which shapes the evolution of star-forming cores. BIMA, OVRO, and the VLA have recently been used to image Orion $\mathrm{KL}$ at very high resolution, and have provided interesting new constraints on the nature of this pivotal massive star forming cloud.

An example of these studies may be found in Figure 1, which presents $1.3 \mathrm{~mm}$ observations of the velocity-integrated emission from $\mathrm{SiO}, \mathrm{C}_{2} \mathrm{H}_{5} \mathrm{CN}$, $\mathrm{HC}_{3} \mathrm{~N}, \mathrm{HNCO}, \mathrm{HCOOCH}_{3}$, and $\mathrm{HDO}$, overlayed on the uniform-weighted dust emission image (from Blake et al. 1996). At a dust temperature of $\mathrm{T}_{\text {dust }}=100 \mathrm{~K}$, the peak column densities of the brightest clumps in the dust map are approximately $1 \times 10^{24} \mathrm{~cm}^{-2}$, leading to high far-IR and midIR opacities of some $\tau \sim 5-10$ at $\lambda=30 \mu \mathrm{m}$. This clumpy distribution leads to a strong mm-wave/IR source anti-correlation, and explains several important features in the molecular line maps. 


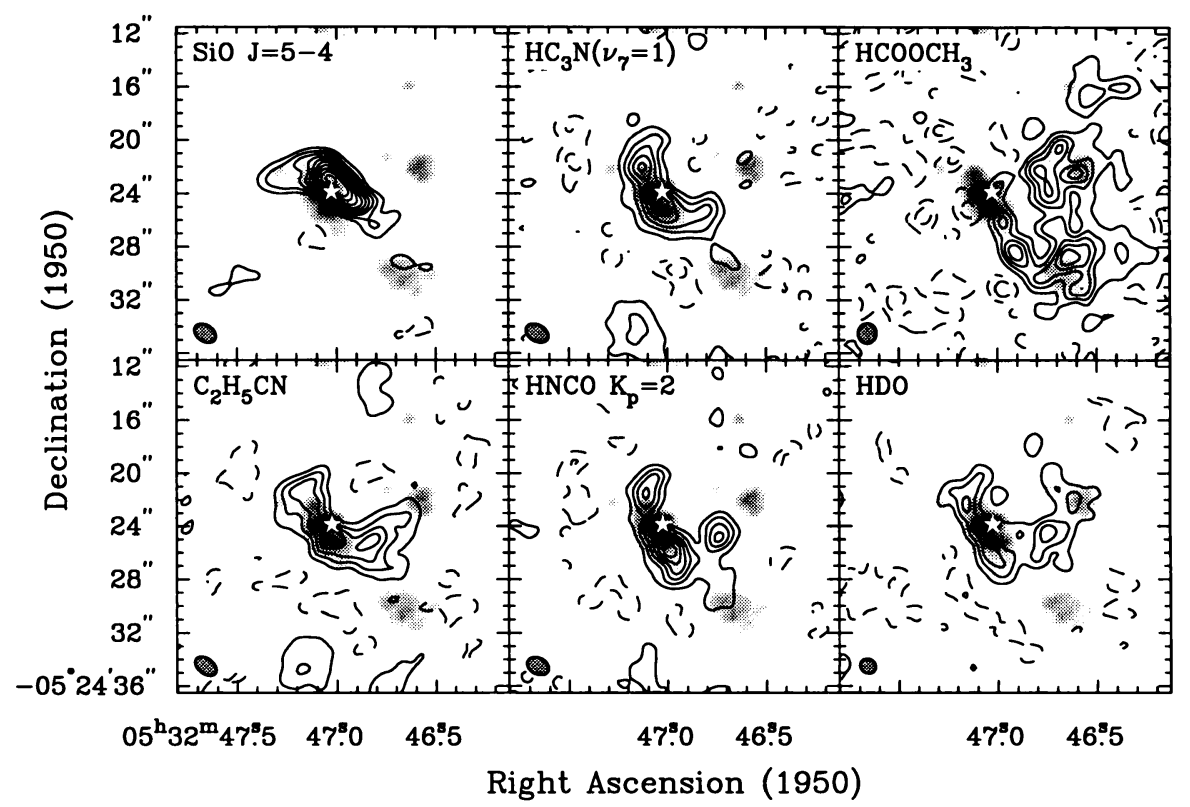

Figure 1. A multi-panel image summarizing various aspects of the chemistry observed toward Orion $\mathrm{KL}$ with the Owens Valley millimeter array at $\lambda=1.3 \mathrm{~mm}$. In each panel, the grey-scale presents the uniform-weighted dust continuum emission at $1 . " 5$ $\times 1 .{ }^{\prime \prime} 0$ resolution. The contours depict the uniform-weighted maps obtained for selected molecules, noted in the upper left hand corner of each panel. Contour levels for the $\mathrm{HCOOCH}_{3} J_{K_{p} K_{o}}=20_{4,17} \rightarrow 19_{4,16} A$ transition are spaced by $0.6 \mathrm{Jy} \mathrm{beam}^{-1}(6 \mathrm{~K})$, those for $\mathrm{SiO} J=5 \rightarrow 4$ at $0.5 \mathrm{Jy}$ beam $^{-1}(8 \mathrm{~K})$, those for HDO $J_{K_{p} K_{o}}=2_{11} \rightarrow 2_{12}$ and HNCO $J_{K_{p} K_{o}}=10_{29} \rightarrow 9_{28}$ at $0.4 \mathrm{Jy} \mathrm{beam}^{-1}(4$ and $7 \mathrm{~K})$, and those for $\mathrm{HC}_{3} \mathrm{~N}\left(\nu_{7}=1\right)$ $J=24 \rightarrow 23(1 f)$ and $\mathrm{C}_{2} \mathrm{H}_{5} \mathrm{CN} J_{K_{p} K_{o}}=24_{2,22} \rightarrow 23_{2,21}$ at $0.3 \mathrm{Jy}$ beam $^{-1}(5 \mathrm{~K})$; contouring begins at the step level in each case. The white star marks the position of radio source I and the $\mathrm{SiO}(v=1)$ maser ring (Blake et al. 1996).

$\mathrm{SiO}$, for example, is known to be strongly depleted in quiescent gas and to preferentially trace energetic processes (see Wright et al. 1983; Bachiller \& Gomez-Gonzales 1992). The OVRO data combined with recent imaging by Chandler \& Depree (1995) and Wright et al. (1995) demonstrate that $\mathrm{SiO}$ is masing even in the ground vibrational state, and that the density in the $v=0$ masing region is $<10^{7} \mathrm{~cm}^{-3}$. The observed velocity fields correlate with those found at extremely high resolution in the $v=1 \mathrm{SiO}$ maser ring surrounding source I near the IRc2 complex (Wright, Plambeck \& Wilner 1996), and the combined images nicely sample the outflow velocity field immediately surrounding the YSO. At greater distances, the emission contours turn approximately $\mathrm{E}-\mathrm{W}$ in a fashion that avoids the regions traced by the $\lambda=1.3 \mathrm{~mm}$ continuum. The broad line wings observed in $\mathrm{SiO}$ 
strongly suggest that shock sputtering and shattering of grains is responsible for the enhanced silicon gas phase abundance, naturally explaining the observed anti-correlation with the dust peaks.

Excitation of $\mathrm{HC}_{3} \mathrm{~N}$ and $\mathrm{HNCO}$ is dominated by (far-)IR photon pumping, thereby offering a means of imaging the far-IR radiation field at high resolution. The $\mathrm{HC}_{3} \mathrm{~N}\left(\lambda_{\text {pump }} \sim 45 \mu \mathrm{m}\right) / \mathrm{HNCO}\left(\lambda_{\text {pump }} \sim 80 \mu \mathrm{m}\right)$ distributions are similar, but not identical. In each case, the emission peaks close to source I, but is coincident neither with source I nor with the $1.3 \mathrm{~mm}$ dust peaks. While the clumps closest to source I are quite similar, on average the HNCO clumps lie at a greater distance, reflecting a softening of the radiation field with increasing extinction.

Finally, emission from $\mathrm{HDO}, \mathrm{C}_{2} \mathrm{H}_{5} \mathrm{CN}$, and $\mathrm{HCOOCH}_{3}$ is presented. These molecules serve as tests of recent hot core chemical models, which rely greatly on the interaction of gas and dust (c.f. Charnley, Tielens \& Millar 1992; Caselli, Hasegawa, \& Herbst 1993). Three kinds of molecules can be distinguished in these models: those species passively trapped in grain mantles and released by heating once star formation occurs (HDO); those synthesized actively on dust grains and then desorbed $\left(\mathrm{C}_{2} \mathrm{H}_{5} \mathrm{CN}\right)$; and those created in the gas phase by combination of ion-molecule and neutral chemistry in the hot core after mantle evaporation $\left(\mathrm{HCOOCH}_{3}\right)$. $\mathrm{HDO}$ also has an overall morphology that is similar to that of $\mathrm{C}_{2} \mathrm{H}_{5} \mathrm{CN}$ and $\mathrm{HC}_{3} \mathrm{~N}\left(\nu_{7}=1\right)$, but a closer inspection of Figure 1 revels that the peaks in the HDO distribution are offset slightly from these two species. Densities of order $10^{9} \mathrm{~cm}^{-3}$ are required to thermalize HDO, and so pumping by far-IR and submillimeter photons are also likely - again suggesting a reddening of the radiation field with distance from source I.

This trend is dramatically outlined by $\mathrm{HCOOCH}_{3}$ for which only a small tongue of emission at the bluest velocities appears near the hot core. Most of the $\mathrm{HCOOCH}_{3}$ emission arises in patterns that avoid the strongest $\mathrm{HDO}$ peaks. Similar features are observed in other complex, oxygen-containing species $\left[\left(\mathrm{CH}_{3}\right)_{2} \mathrm{O}, \mathrm{H}_{2} \mathrm{CO}\right.$, also $\left.\mathrm{H}_{2} \mathrm{CS}\right]$. In each case, the bulk of the emission is centered close to a dust clump on the side toward source I. Such relationships among the more complex molecules are predicted by ion-molecule networks (Charnley et al. 1992), chemistry not favored by the high densities and temperatures immediately adjacent to massive YSOs.

Thus, the observed spatial patterns suggest that variations in both the physical conditions and grain mantle composition play important roles in the chemical evolution of the Orion core, and confirm the dominant role of the source I/IRc2 complex, which appears to lie close to and behind the dense clumps, whose exteriors are currently being evaporated and/or ablated. In effect, these cool cores serve as reservoirs for the chemically and spectrally rich hot core phase of massive star formation first recognized in 
Orion, and help to maintain it for the $\sim 10^{4-5}$ years required for the chemistry to reach the observed level of complexity. Furthermore, the reddening of the source I radiation field with distance may lead to efficient heating of the clump exteriors in "cool" photon dominated regions similar to those recently proposed to exist around low-mass YSOs (Spaans et al. 1995) but not to extensive dissipation of the dense gas, naturally forming the warm cocoons of gas that may help resolve the lifetime paradox associated with ultracompact HII regions (Depree, Rodriguez \& Goss 1995).

\section{The observational characterization of low mass YSOs}

The same tracers used to study chemical processes in high mass starforming cores have also been widely applied to the study of isolated, low mass YSOs. From observational studies and theoretical models, a number of environments, characterized by distinct differences in their physical conditions but with overlapping spatial extents, have been enumerated. A number of observations have been carried out whose central theme is to discover whether certain species can be found that selectively trace the region(s) of interest. We discuss each in turn below.

\subsection{OUTFLOWS}

One of the earliest, and initially puzzling, observational signposts of star formation in molecular clouds was the widespread detection of outflowing, supersonic gas (Snell, Loren \& Plambeck 1980). That the ejection of material at high speeds from deep in the gravitational well of young stars is a necessary compliment to mass accretion is now thought to arise naturally from the need to shed the vast majority of the original angular momentum content of collapsing cloud cores. By ejecting a small amount of matter to large distances at high velocities, outflows efficiently transfer angular momentum outward while allowing mass accretion to occur.

Despite the general agreement as to their importance, the means by which the YSO/accretion disk system produces and directs the outflows is still much debated (Pelletier \& Pudritz 1992; Shu et al. 1995). Imaging and kinematic studies can, in principle, constrain the intrinsic properties of the ejection process. High resolution optical images of the forbidden line emission from $\mathrm{T}$ Tauri stars often delineate narrow jets moving at speeds of up to $400-500 \mathrm{~km} \mathrm{~s}^{-1}$, whose transverse width is comparable to the spatial resolution employed (see, for example, Heathcote et al. 1996). Observations of many YSOs in different evolutionary states are needed to assess the degree to which the collimation is inherent to the jet, externally forced, or an observational artifact. 
Such studies should include the very earliest stages of star formation that are difficult to examine optically due to the tremendous extinction present. Millimeter-wave telescopes of the type first used to discover molecular outflows often reveal outflows with opening angles that are substantially larger than those inferred from optical emission line images. The spatial resolution of most studies is rather poorer than those at visible wavelengths, but it has been suggested that the very youngest sources have well collimated molecular outflows which evolve over time to produce the more poorly collimated flows observed in older YSOs (Bachiller \& GomezGonzales 1992; André, Ward-Thompson \& Barsony 1993).

Of the many sources that have been studied, outflows in the NGC1333 molecular cloud are particular amenable to study at (sub) $\mathrm{mm}$ wavelengths. NGC 1333, located in the Perseus molecular cloud complex, is a site of active low- and intermediate-star formation, which has been going on for only the last 100,000 years. IR and (sub)mm continuum and line observations have revealed NGC 1333 to be exceptionally active, with a multitude of IR sources and at least five distinct outflows in a compact 100 square arc minute area (Sandell et al. 1994, Warin et al. 1996). Indeed, the cloud structure appears to be completely dominated by the outflows which snake thru the entire region. The core of NGC 1333 may well be undergoing sequential star formation, perhaps forming a loosely bound cluster, that is driven by winds sweeping up material and shock compressing the gas. Understanding the immediate fate of the cloud, disruption or continued star formation, requires better tracers of the outflow/cloud interface(s).

Combined interferometric and single dish mapping has demonstrated that the outflows impact dense, cold pre-shock gas $\left(n\left(\mathrm{H}_{2}\right)=10^{5} \mathrm{~cm}^{-3}\right.$, $T=20 \mathrm{~K})$ that is subsequently heated and compressed $\left(n\left(\mathrm{H}_{2}\right)=10^{7} \mathrm{~cm}^{-3}\right.$, $T=100 \mathrm{~K})$ (Blake et al. 1995). This results in extraordinarily strong molecular emission from the post-shocked gas. Consider the case of the molecular bow shock near IRAS 2, an overview of which is presented in Figure 2. First discovered by Sandell et al. (1994) at the tip of a CO outflow that was unresolved in the transverse dimension at $15^{\prime \prime}$ resolution, this source appears to provide a molecular counterpart to the optical jets so frequently observed in pre-main sequence objects.

Of the two cores traced by the CS emission at the right edge of Figure 2 (IRAS2A and IRAS2B), the jet-like structure traced by the high velocity $\mathrm{SiO}$ emission clearly projects back toward only IRAS2A some 17,400 AU distant. While the jet itself is optically invisible, its diameter is constrained to be $\leq 500 \mathrm{AU}$ by the $\mathrm{SiO}$ and $\mathrm{CS}$ images, which also highlight the importance of the ambient medium in shaping the flow. For example, the low velocity $\mathrm{CS}$ is aligned primarily along a $\mathrm{N}-\mathrm{S}$ ridge that is distorted into a bow-shock shape by the outflow, which is remarkably similar to numerical 


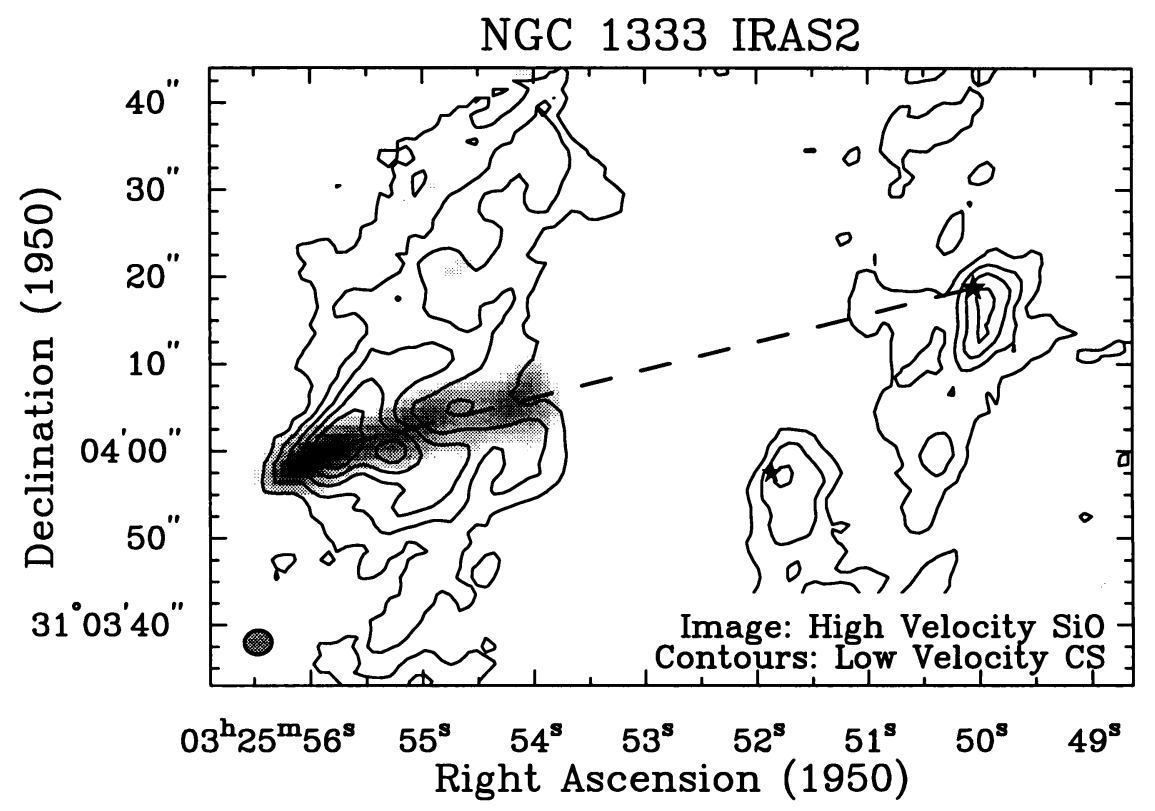

Figure 2. OVRO image of the CS J=2-1 emission from the ambient gas (contours) and $\mathrm{SiO} \mathrm{J}=2-1$ emission from high velocity gas (greyscale) associated with the outflow from NGC 1333 IRAS 2. The "stars" mark the positions of the IRAS $2 A$ and $2 B \lambda=2.7 \mathrm{~mm}$ continuum sources. The $\mathrm{SiO}$ emission traces the extremely collimated outflow while the CS emission traces the outflow interaction region within the cloud as well as the molecular cores associated with IRAS $2 \mathrm{~A}$ and $2 \mathrm{~B}$.

simulations of jets driving into increasing density gradients (de Gouveia Dal Pino, Birkinshaw \& Benz 1996). Finally, the large spatial extent of the molecular emission along the jet offers a rare opportunity to examine in detail the chemistry of dense, shocked interstellar gas.

In this source, and in the nearby outflow from IRAS 4A, the abundances of many species, both refractory and volatile $\left(\mathrm{SiO}, \mathrm{SO}, \mathrm{CS}, \mathrm{HCN}, \mathrm{H}_{2} \mathrm{CO}\right.$, $\mathrm{CH}_{3} \mathrm{OH}$ ), are considerably enhanced, as they are in many other outflows (see Bachiller in this volume, Blake et al. 1995). As might be expected, spatial offsets are observed between the refractory (e.g. SiO) species produced in shocked material and more volatile species $\left(\mathrm{HCN}, \mathrm{CH}_{3} \mathrm{OH}\right)$ that can, in principle, be produced by sputtering or desorption of grain mantles. Thus, much like the hot cores surrounding massive YSOs, the chemical composition of outflow/cloud interaction zones may be used to estimate the products of grain mantle chemistry. 


\subsection{THE DYNAMICALLY ACCRETING ENVELOPE}

While observations with single dish (sub)mm-wave telescopes have spatial resolutions that are too coarse to detect molecular emission from circumstellar disks, they provide excellent probes of the simultaneous ability of infall and outflow to modify the global YSO environment (Myers et al. 1996; Zhou 1995). Indeed, the tremendous frequency versatility of the new generation of large-aperture (sub) $\mathrm{mm}$ telescopes and arrays enable the general chemical composition of and physical conditions in the dynamically infalling envelopes surrounding YSOs to be examined in detail.

For example, in extensive observations of deeply embedded sources in the Ophiuchus (IRAS 16293-2422; Blake et al. 1994, van Dishoeck et al. 1995) and Serpens molecular clouds (S68FIRS1; McMullin et al. 1994), distinct kinematic features and enhanced abundances patterns were noticed for non-volatile "energy rich" species such as $\mathrm{SiO}$ and $\mathrm{SO}_{2}$ as contrasted to the chemistry of volatile molecules (e.g. $\mathrm{HCN} / \mathrm{HNC}, \mathrm{CH}_{3} \mathrm{OH}, \mathrm{H}_{2} \mathrm{CO}$ ), where a more complex picture emerges. In the volatiles, both enhancements and depletions in abundances were observed in the dense material. A rough correspondence of the species expected to be produced $\left(\mathrm{CH}_{3} \mathrm{OH}, \mathrm{H}_{2} \mathrm{CO}\right)$ or consumed (HNC, $\mathrm{CCH}$ ) by grain chemistry was found (Charnley, Tielens \& Millar 1992; Caselli, Hasegawa \& Herbst 1993).

Sources such as IRAS4A which drive the Perseus outflows outlined above show similar abundance patterns, but with truly dramatic quantitative swings. In quiescent gas surrounding the young stars, kinematic signatures consistent with dynamic accretion are revealed, and depletion factors of 25-50 are found for all molecules, including CO (Blake et al. 1995)! At velocities well removed from systemic, we observed large enhancements of both non-volatile $\left(\mathrm{SiO}, \mathrm{SO}, \mathrm{SO}_{2}\right)$ and fragile organic $\left(\mathrm{H}_{2} \mathrm{CO}\right.$ and $\left.\mathrm{CH}_{3} \mathrm{OH}\right)$ species. This pattern is consistent with a picture wherein the early stellar stages are marked by a "freezing" out of molecules onto grain surfaces; later in the evolution, as the stellar outflow and photon flux heat the gas and dust, molecules evaporate from the grain surfaces rejoining the gas phase (Bergin, Langer \& Goldsmith 1995; Willacy, Rawlings \& Williams 1994; Pineau des Forêts, Flower \& Millar 1991).

Less embedded sources show similar characteristics. Single dish and interferometric imaging of a suite of YSOs in Taurus, for example, shows strong emission from $\mathrm{CO}$ and $\mathrm{HCO}^{+}$, but weak emission from a variety of other species (Hogerheijde et al. 1997a,b). Images of three of these objects are shown in Figure 3. The combined line and continuum data can only be fit by density distributions which lack an intrinsic size scale and which are sufficiently centrally condensed. A lower bound on the fractional ionization in the infall envelopes is derived via the average $\mathrm{HCO}^{+}$abundance of 


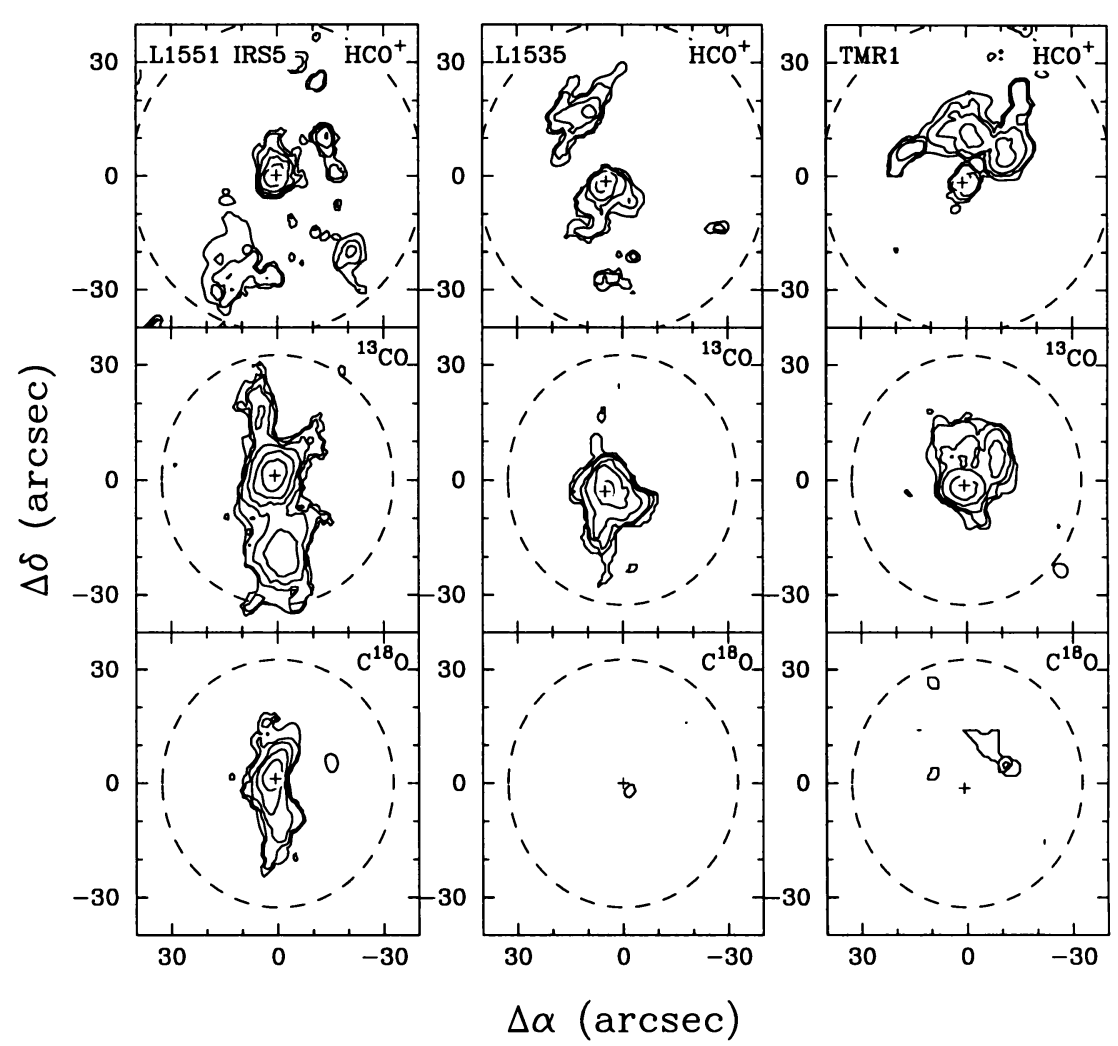

Figure 3. Maps of the velocity integrated intensity of $\mathrm{HCO}^{+} 1-0$ (top), ${ }^{13} \mathrm{CO} 1-0$ (middle), and $\mathrm{C}^{18} \mathrm{O} 1-0$ (bottom) from L1551, L1535, and TMR1. The dashed circle indicates the OVRO primary beam, while the cross shows the best fit continuum position. The beam sizes are approximately $6^{\prime \prime} \times 6^{\prime \prime}$, and the contour levels start at 4.8 times the RMS level (approximately $70 \mathrm{mJy} /$ beam and $150 \mathrm{mJy} /$ beam for $\mathrm{HCO}^{+}$and ${ }^{13} \mathrm{CO} / \mathrm{C}^{18} \mathrm{O}$ ), increasing logarithmically (Hogerheijde et al. 1997b).

$\sim 1 \times 10^{-8}$. Overall, the results of this survey are quite consistent with the emerging theoretical picture of star formation, and highlight the important role of outflow/envelope interactions in that a close connection between the outflow(s) and envelope structure is found.

\subsection{CIRCUMSTELLAR DISKS}

Two important parameters in models of the protosolar nebula and of protoplanetary nebulae are the radial extent of the disk and the variation of surface density with radius. Simple conservation of angular momentum 


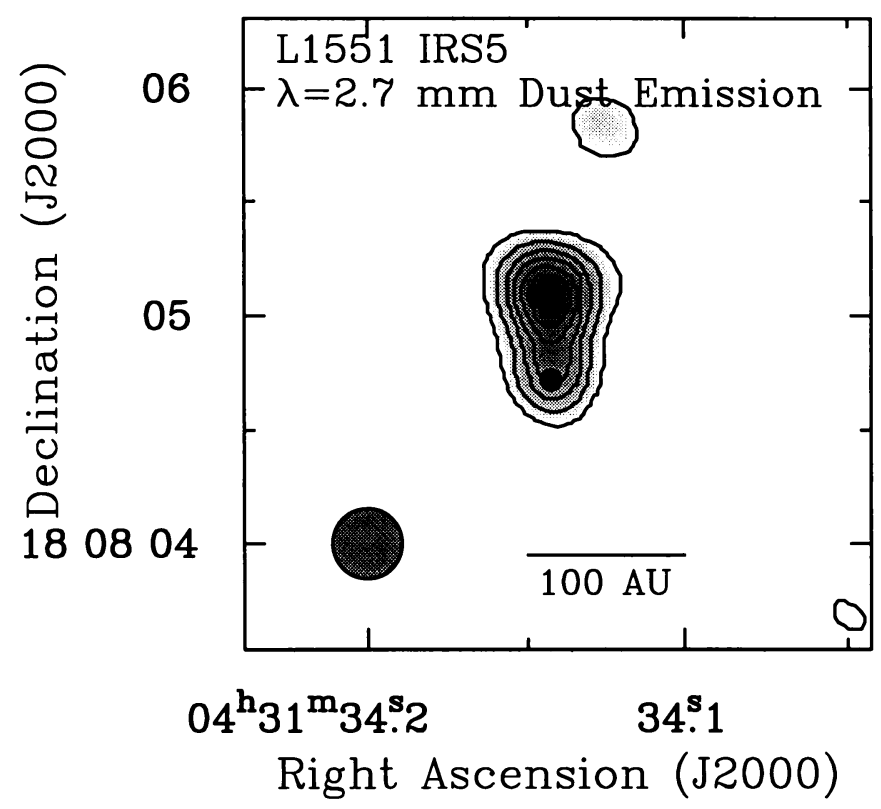

Figure 4. BIMA $\lambda=2.7 \mathrm{~mm}$ continuum map, shown in contours and greyscale, of the L1551 IRS5 system (Looney et al. 1996). The dots mark the positions of the two $\lambda=2 \mathrm{~cm}$ emission regions (Rodriguez et al. 1986). The positions are coincident with the $\lambda=2.7 \mathrm{~mm}$ emission, suggesting that L1551 IRS5 is a binary system with dust around each star rather a single star plus disk.

suggests that these disks should be of order 10-100 AU in radius. In more detailed modeling the extent of the disk and distribution within the disk are related to the viscosity and angular momentum transport, which are only beginning to be understood (c.f. Adams \& Lin 1993). Observationally, the situation has not been much more precise. Maps of molecular emission from a number of systems noted above reveal flattened structures with radii of 1000-3000 AU but millimeter continuum maps of the same system show much more compact, often unresolved, emission (Sargent 1996).

Developments in (sub) $\mathrm{mm}$ instrumentation now allow astronomers to measure, or set stricter limits on, the disk properties in YSOs. For example, HL Tau and L1551 IRS5 have now been imaged with roughly $0 . " 5$ and $0 . " 3$ resolution (Lay et al. 1994; Mundy et al. 1996; Looney et al. 1996). In HL Tau, the $\lambda=2.7 \mathrm{~mm}$ emission reveals an elongated circumstellar disk that is obscuring the star in the optical and near-IR. From model fitting of the emission at a variety of wavelengths, an outer radius for the disk of 90 to $160 \mathrm{AU}$ is derived, and the surface density is found to fall off as $r^{0}$ to $r^{-1}$. Such a radial dependence in the surface density is shallower than the $r^{-1.5}$ 
power-law usually posited for the early solar nebula.

In L1551 IRS5, the $\lambda=2.7 \mathrm{~mm}$ emission is resolved (see Figure 4, Looney et al. 1996) into two sources which are coincident with the two sources previously seen at $\lambda=2 \mathrm{~cm}$ (Rodriguez et al. 1986). From this work it has been proposed that L1551 IRS5 is actually a binary system, with circumstellar disks of radii $<50 \mathrm{AU}$ associated with the individual stars. This is a complete change in the paradigm for L1551 IRS5 which was previously thought to be a single star with 50-100 AU disk (Keene \& Masson 1990, Lay et al. 1994), and is an important reminder of the fact that most low mass YSOs are likely to be multiple systems with separations that cannot be easily resolved with current instrumentation.

Observations with the OVRO array have concentrated on studies at $\lambda=1.3 \mathrm{~mm}$ where the highest resolution and best sensitivity to dust emission can be achieved. Images of the deeply embedded NGC 1333 IRAS 4A and 4B systems have confirmed the binary nature of IRAS 4A (separation $1.8^{\prime \prime}$ or $400 \mathrm{AU}$ (Lay, Carlstrom \& Hills 1995)) and find that most of the emitting material in these young systems is still in 1000 AU-sized envelopes rather than in circumstellar disks (Mundy et al. 1997). Initial 0."7 resolution OVRO results near $\nu=267 \mathrm{GHz}$ for T Tau show, surprisingly, a compact circumstellar disk around T Tau N (van Langevelde et al. 1996) and not T Tau S as expected from previous observations (Ghez et al. 1991).

To date, such sub-arcsecond continuum imaging has achieved sensitivities in the few to few 10's of mJy range after several transits. While sufficient to detect and map the dust emission from many disks, the corresponding brightness temperatures in spectral channels narrow enough to be kinematically useful are still 100-300 K. This is considerably brighter than limits on the dust and expected gas temperatures at $\geq 30 \mathrm{AU}$, and so substantially larger arrays such as the MMA will be needed for high dynamic range imaging of the gas in YSO accretion disks at $0 .{ }^{\prime \prime} 1-0 .{ }^{\prime \prime} 3$ resolution. Initial efforts at more modest resolution $\left(1^{\prime \prime}-2^{\prime \prime}\right)$ have concentrated on the $\mathrm{CO}$ molecule, and have led to very high detection rates of $\mathrm{CO}(2-1)$ emission from disks around optically visible T Tauri stars (Sargent \& Koerner 1997). These and similar studies from the BIMA, Nobeyama, and Plateau de Bure interferometers are among the first to directly image the bulk material distribution in the 50-200 AU scale around the full evolutionary range of Sun-like stars. In the future we can expect far more detailed studies of the chemical composition of circumstellar disks, the results of which can be compared to theoretical models and the chemistry in primitive solar system objects such as comets, meteorites, and icy satellites. 


\section{Acknowledgements}

Much of the research described in this review is the result of an active collaboration between groups at Leiden, Maryland, and Caltech. The author gratefully acknowledges the efforts of Ewine van Dishoeck and Lee Mundy, along with those of their colleagues, in carrying forward the observations described herein.

\section{References}

Adams, F.C., Lin, D.C. 1993, in Protostars \& Planets III, E.H. Levy, J. Lunine, eds. (Univ. Arizona Press, Tucson), p. 721.

André, P., Ward-Tompson, D., Barsony, M. 1993, ApJ, 206, 122.

Bachiller, R., Gomez-Gonzalez, J. 1992, A\&AR, 3, 257.

Beckwith, S.V.W. et al. 1990, AA, 286, 149.

Beckwith, S.V.W., Sargent, A.I. 1996, Nature, 383, 139.

Bergin, E.A., Langer, W.D., Goldsmith, P.F. 1995, ApJ, 441, 222.

Blake, G.A., Sutton, E.C., Masson, C.R., \& Phillips, T.G. 1987, ApJ, 315, 621.

Blake, G.A., van Dishoeck, E.F., Jansen, D., Groesbeck, T. \& Mundy, L.G. 1994, ApJ, 441,689 .

Blake, G.A., Sandell, G., van Dishoeck, E.F., Groesbeck, T., Mundy, L.G., Aspin, C. 1995, ApJ, 441, 689.

Blake, G.A., Mundy, L.G., Carlstrom, J., Padin, S., Scott, S.L., Scoville, N.Z., Woody, D.P. 1996, ApJ, 472, L49

Caselli, P., Hasegawa, T.I., \& Herbst, E. 1993, ApJ, 408, 548.

Chandler, C.J., Carlstrom, J.E., Scoville, N.Z., Dent, W.R.F., Geballe, T.R. 1993, ApJ, 412, L71.

Chandler, C.J. \& Depree, C.G. 1995, ApJ, 455, L67.

Charnley, S.B., Tielens, A.G.G.M., Millar, T.J. 1992, ApJ, 399, L71.

de Gouveia Dal Pino, E.M., Birkinshaw, M., Benz, W. 1996, ApJ, 460, L111.

Depree, G.G., Rodriguez, L.F., Goss, W.M. 1995, RMAA, 31, 39.

Dougados, C., Léna, P., Ridgway, S.T., Christou, J.C., Probst, R.G. 1993, ApJ, 406, 112.

Dutrey, A., Guilloteau, S., Simon, M. 1994, AA, 286, 149.

Genzel, R., Stutzki, J. 1989, ARAA, 27, 41.

Ghez, A. et al. 1991, AJ, 102, 2066.

Hartquist, T.W., Rawlings, J.M.C., Williams, D.A., Dalgarno, A. 1993, QJRAS, 34, 213.

Heathcote, S., Morse, J., Hartigan, P, Reipurth, B., Schwartz, R.D., Bally, J., Stone, J.M. 1996, AJ, 112, 1141.

Hogerheijde, M.R., van Dishoeck, E.F., Blake, G.A., van Langevelde, H.J. 1997a, ApJ, submitted.

Hogerheijde, M.R., van Dishoeck, E.F., Blake, G.A., van Langevelde, H.J. 1997b, ApJ, in preparation.

Johansson, L., Andersson, C., Elldér, J, Friberg, P., Hjalmarson, Å. 1984, AA, 130, 227.

Keene, J., Masson, C.R. 1991, ApJ 355, 635

Lacy, J.H., Carr, J.S., Evans, N.J., Baas, F., Achtermann, J.M., Arens, J.F. 1991, ApJ, $376,556$.

Lay, O., Carlstrom, J., Hills, R.E., Phillips, T.G. 1994, ApJ, 434, L75.

Lay, O., Carlstrom, J., Hills, R.E. 1995, ApJ, 452, L73.

Li, Z.Y., Shu, F.H. 1996, ApJ, 468, 261.

Looney, L.W., Mundy, L.G., Welch, W.J. 1996, ApJ, in press.

Marcy, G.W., Butler, R.P. 1996, ApJ, 464, L147.

Menten, K.M., Reid, M.J. 1995, ApJ, 445, L157.

Masson, C.R., Chernin, L.M., 1993, Ap. J., 414, 230. 
McMullin, J.P., Mundy, L.G., Wilking, B., Hezel, T., Blake, G.A. 1994, ApJ, 424, 222. Mundy, L.G., et al. 1996, ApJ, 464, L169.

Mundy, L.G. et al. 1997, ApJ, submitted.

Myers, P.C., Mardonnes, D., Tafalla, M., Williams, J.P., Wilner, D.J. 1996, ApJ 465, L133

Pelletier, G., Pudritz, R.E. 1992, Ap. J. 493, 117.

Pineau des Forêts, G., Flower, D.R., Millar, T.J. 1991, MNRAS, 253, 217.

Plambeck, R.L., Wright, M.C.H., Welch, W.J., Bieging, J.H., Baud, B., Ho, P., Vogel, S.N. 1982, ApJ, 259, 617.

Plambeck, R.L., Wright, M.C.H., Mundy, L.G., Looney, L.W. 1995, ApJ, 455, L189.

Rodriguez, L.F., Cantó, J., Torrelles, J., Ho, P.T.P. 1986, ApJ, 301, L25.

Sandell, G., Knee, L.B.G., Aspin, C., Robson, I.E., Russell, A.P.G., 1994, AA, 285, L1.

Sargent, A.I., Welch, W.J. 1995, ARAA, 31, 297.

Sargent, A.I. 1996, in Disks and Outflows around Young Stars, S.V.W. Beckwith, J. Staude, eds. (Springer-Verlag, Berlin)

Sargent, A.I., Koerner, D.W. 1997, in preparation.

Shu, F.H. 1995, Rev.Mex.AA(Serie de Conf.), 1, 375.

Shu, F.H., Najita, J., Ostriker, E.O., Shang, H. 1995, ApJ, 455, L155.

Snell, R.L., Loren, R.B., Plambeck, R.L. 1980, ApJ, 239, L17.

Spaans, M., Hogerheijde, M., Mundy, L., van Dishoeck, E. 1995, ApJ, 455, L167.

van Dishoeck, E.F., Blake, G.A., Draine, B.T., Lunine, J.I. 1993, in Protostars \& Planets III, E.H. Levy, J. Lunine, eds. (Univ. Arizona Press, Tucson), p. 163.

van Dishoeck, E.F., Blake, G.A., Jansen, D., Groesbeck, T. 1995, ApJ, 447, 760.

van Langevelde et al. 1996, in IAU Symposium 170, CO: Twenty-five Years of Millimeter wave Spectroscopy, ed. W.B. Latter et al. (Dordrecht:Kluwer).

Warin, S., Castets, A., Langer, W.D., Wilson, R.W., Pagani, L. 1996, AA, 306, 935.

Willacy, K., Rawlings, J.M.C., Williams, D.A. 1994, MNRAS, 269, 921.

Wright, M.C.H., Plambeck, R.L., Vogel, S.N., Ho, P. Welch, W.J. 1983, ApJ, 267, L41.

Wright, M.C.H., Plambeck, R.L., Mundy, L.G., \& Looney, L.W. 1995, ApJ, 455, L185.

Wright, M.C.H., Plambeck, R.L., Wilner, D.J. 1996, ApJ 469, 216

Wynn-Williams, C.G., Genzel, G., Becklin, E.E., Downes, D. 1984, ApJ, 281, 172.

Zhou, S. 1995, ApJ, 442, 685.

\section{Discussion}

Pecker: About GL2591, you said you could determine both column densities and abundances. This puzzles me, in view of the highly inhomogeneous structure along the line of sight. I understand how you can get column density, but I feel that going to abundances would require knowledge of the characteristics of the inhomogeneous medium. How do you get them?

Blake: You get them by obtaining very high velocity resolution observations $(\Delta V \leq 10 \mathrm{~km} / \mathrm{s})$ which in many cases reveal cool and warm gas to be kinematically distinct. A good example of this approach is provided by the infrared CO observations of Mitchell et al. (1989, ApJ 341, 1020) toward GL 2591, who find well resolved absorption arising from cool $(\sim 38 \mathrm{~K})$ and warm $(\sim 200 \mathrm{~K})$ molecular gas. 